\title{
An investigation of quality improvement initiatives in decreasing the rate of avoidable 30-day, skilled nursing facility-to-hospital readmissions: a systematic review
}

\author{
This article was published in the following Dove Press journal: \\ Clinical Interventions in Aging \\ 25 January 2017 \\ Number of times this article has been viewed
}

\section{Michael Mileski \\ Joseph Baar Topinka \\ Kimberly Lee \\ Matthew Brooks \\ Christopher McNeil \\ Jenna Jackson}

School of Health Administration, Texas State University, San Marcos, TX, USA
Correspondence: Michael Mileski Texas State University, 60I University Drive, San Marcos, TX 78666, USA

Tel + I 8327524419

Email mileski@txstate.edu
Objectives: The main objective was to investigate the applicability and effectiveness of quality improvement initiatives in decreasing the rate of avoidable 30-day, skilled nursing facility (SNF)-to-hospital readmissions.

Problem: The rate of rehospitalizations from SNF within 30 days of original discharge has increased within the last decade.

Setting: The research team participants conducted a literature review via Cumulative Index of Nursing and Allied Health Literature and PubMed to collect data about quality improvement implemented in SNFs.

Results: The most common facilitator was the incorporation of specialized staff. The most cited barriers were quality improvement tracking and implementation.

Conclusion: These strategy examples can be useful to acute care hospitals attempting to lower bounce back from subacute care providers and long-term care facilities seeking quality improvement initiatives to reduce hospital readmissions.

Keywords: readmissions, skilled nursing, quality improvement, hospital, finance, Medicare, transfers

\section{Introduction}

Unnecessary hospital readmissions from skilled nursing facilities (SNFs) are gaining attention as a quality improvement focus due to their financial expense and association with increased patient complications. ${ }^{1}$ Approximately $20 \%-25 \%$ of patients discharged to SNFs are readmitted to acute care hospitals within 30 days of discharge in the USA. $^{2}$ The average Medicare payment per rehospitalization was $\$ 10,352$ in 2006, with hospital readmissions accounting for $\sim \$ 4.34$ billion in reimbursement expenses. Transfers between hospitals and SNFs are associated with increased medical errors and patient stress that can lead to functional and mental decline. ${ }^{3}$

The rate of rehospitalizations from SNFs within 30 days of original discharge has increased to nearly one-fifth since 2004 , and $\sim 90 \%$ of these rehospitalizations were unplanned. ${ }^{2}$ Hospitals contributed to this trend by discharging higher-acuity patients in order to reduce average length of stay and to improve patient throughput, resulting in better financial margins. ${ }^{4}$ This practice caused many nursing homes that once focused on older, noncognitive, and functionally deficient residents to shift more care toward shorter-term, high-acuity, and medically complex patients. Rehospitalization is not 
always avoidable for this population; however, retrospective analysis of readmission diagnoses and variation in geographic readmission rates suggest this metric can be improved. ${ }^{5}$

The Medicare Payment Advisory Commission found that the five potentially avoidable conditions - congestive heart failure, urinary tract infection, respiratory infection, sepsis, and electrolyte imbalance - accounted for $78 \%$ of all 30 -day readmissions from SNFs to hospitals..$^{5}$ The average rate of readmissions among SNFs also shows wide geographic variations, with a low of $15.1 \%$ in Utah and a high of $28.2 \%$ in Louisiana. ${ }^{2}$ Some of the state level differences can be attributed to reimbursement incentives, for example, a $\$ 10$ higher Medicaid payment rate being associated with a $9 \%$ lower odds of hospitalization. ${ }^{6}$

Currently, a SNF that reduces 30-day rehospitalization rates primarily generates savings for Medicare because Medicare Part A pays full cost of the first 20 days of SNF room and board and covers hospitalization expenses. ${ }^{6}$ After day 21 in a SNF, there is a lower cost to Medicare as the patient now has some responsibility for their own care via copayments required by Medicare. ${ }^{7}$ The lack of compensation from Medicare for the amount of care needed has created a financial incentive for SNFs to not initiate quality improvement programs. States with higher Medicaid SNF reimbursement rates generally have lower hospital readmission rates because they have more financial incentive to keep complex care patients at the facility. The Affordable Care Act (ACA) began to change the payment incentives that promote this behavior in October $2012 .^{8}$

One of the programs established under the ACA was the Hospital Readmissions Reduction Program, which reduced reimbursement under the Prospective Payment System for hospitals with excess 30-day readmission for some diagnoses. ${ }^{8}$ Hospitals are incentivized to not discharge patients too early and to identify and coordinate with SNFs that can maintain low readmission rates. Increased scrutiny from the Centers for Medicare and Medicaid Services (CMS) and collaboration between hospitals and SNFs led to a focus on providing high quality care at reduced costs. ${ }^{9}$

The ACA promoted the testing of a number of pay-forperformance (PFP) schemes. For instance, the ACA called for bundling payments for episodes of care and value-based reimbursement. This effort was made in order to collect data on the feasibility of CMS programs that could improve patient care without increasing cost. ${ }^{10}$ The ongoing Nursing Home Value-Based Purchasing Demonstration, which started in July 2009, gives participating SNFs in Arizona, New York, and Wisconsin financial incentives to lower avoidable hospitalization rates. ${ }^{7}$ Participating SNFs can earn a PFP incentive if they are within the top $20 \%$ of SNFs or if they are in the top $20 \%$ of SNFs for overall improvement. The main objective of this literature review was to investigate the applicability and effectiveness of quality improvement initiatives in decreasing the rate of avoidable 30-day, SNF-to-hospital readmissions. It seems that most quality improvement initiative (QII) studies utilized in this review showed some element of success in their implementation. This study will look further into these successes and also identify areas where barriers also existed to meeting them.

\section{Materials and methods Design}

This study used a systematic review of peer-reviewed articles found in indexed databases. The Preferred Reporting Items for Systematic Reviews (PRISMA) guidelines were used to ensure consistent and precise reporting of results. The initial search was conducted on June 20, 2016 and final search was completed on July 10, 2016 using the Cumulative Index of Nursing and Allied Health Literature and PubMed. The Medical Subject Headings at the National Center for Biotechnology Information were used to discover keywords for the queries. However, there were multiple terms that appeared for the two acronyms searched PFP and SNF. To avoid artificially narrowing the initial search scope, a more inclusive keyword search was utilized. The authors examined the search results to taper the final scope. Understanding the most commonly revealed keywords led to the complex, four-string Boolean search included in Figure 1. A review of articles found in the citations led to additional resources that met the inclusion criteria.

\section{Inclusion criteria}

All authors individually reviewed the articles from the search, determined germane literature, and summarized themes. Inclusion criteria included English-language and peer-reviewed articles published by academic journals or universities between January 1, 2009 and July 10, 2016. Articles had to explore at least one quality improvement strategy for reducing 30-day hospital readmission rates from SNFs to hospitals. Articles could reference preventable readmission rates or total readmission rates within the 30-day time period.

\section{Exclusion criteria}

Articles were only incorporated if deemed germane by all authors. Trade industry reports and poster presentations without clear, scientific format, and a peer review process were excluded. Articles that dealt with discharge from SNFs 
CINAHL complete

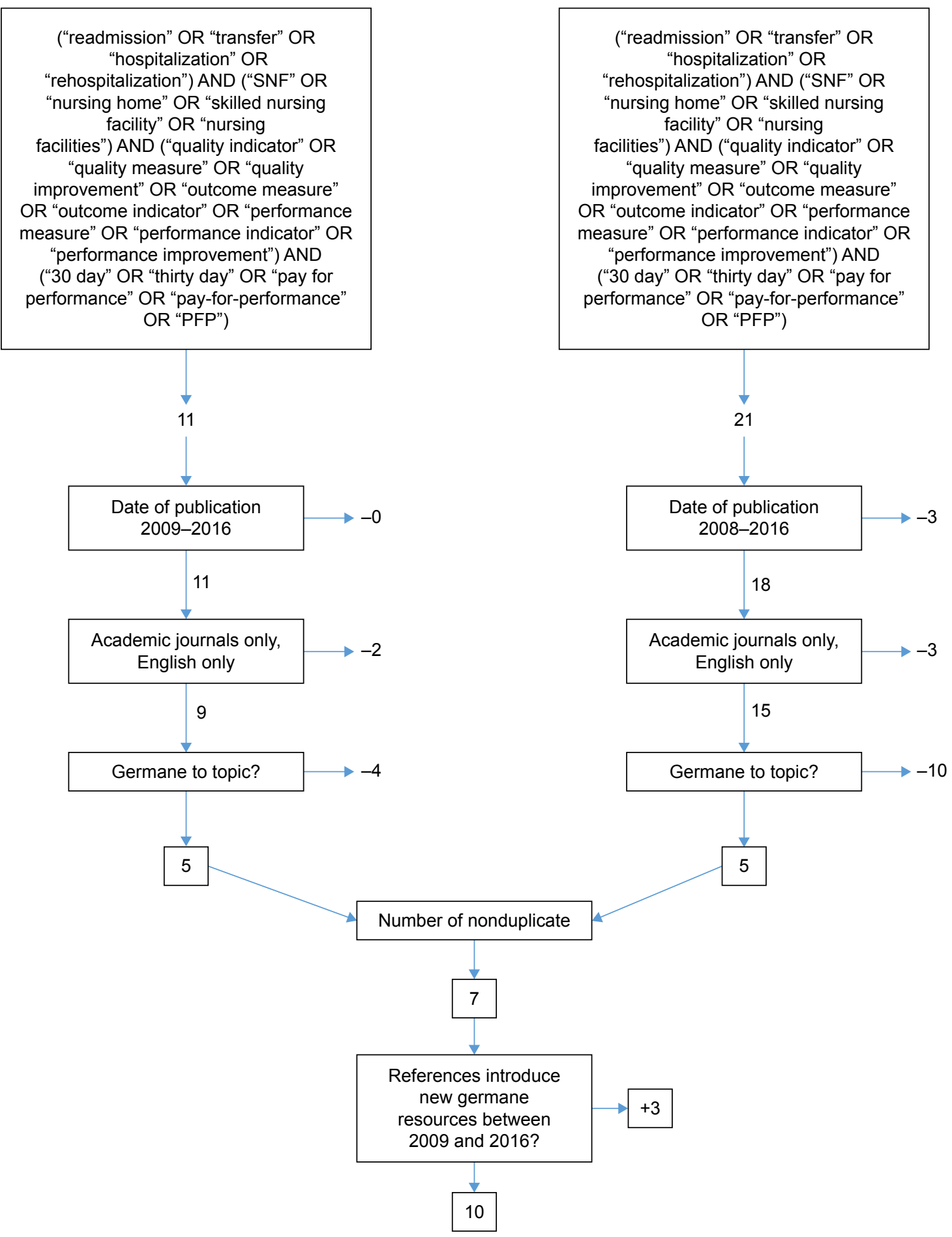

Figure I Preferred Reporting Items for Systematic Reviews flow diagram.

Abbreviations: CINAHL, Cumulative Index of Nursing and Allied Health Literature; PFP, pay-for-performance; SNF, skilled nursing facilities.

or hospitals-to-home settings were not included. Articles that only mentioned readmission rates without reference to the efficacy of quality improvements were not included. Articles that did not mention rates for 30-day readmission were excluded. Similar examination outcomes demonstrated that the authors had a parallel understanding of the research subject and scope. Bias was not considered when reviewing the research involved in this study. The final sample of articles after meeting exclusion criteria was then analyzed further for consensus among all authors for final inclusion. When analyzed, this sample yielded a kappa statistic $(\mathrm{k}=1)$, showing strong reliability. 


\section{Data analysis}

Narrative summaries related to factors that influenced quality improvement initiatives were extracted from each article. These, in turn, were grouped into larger recurring themes that were either key determinants or impediments to success. The themes chosen were by consensus of the authors. Those chosen were agreed upon to be ones which provided overarching summary to the facilitators and barriers extracted. These themes were then divided in two affinity matrix tables for facilitators and barriers. Each table documents the themes, their citation occurrence, their frequency sum, and frequency percentage.

\section{Results}

\section{Study selection}

The article selection process is outlined in the PRISMA flow diagram in Figure 1. The initial search protocol identified a total of 32 articles from the database, of which 26 were nonduplicate. Nineteen articles were excluded for not meeting the predetermined inclusion requirements, leaving seven articles remaining. A further analysis of citations generated three additional articles that met the inclusion requirements. A total of 10 articles were used for qualitative analysis.

\section{Assessment tools}

The 10 articles included in the qualitative analysis are summarized in Table 1 from most recent to oldest.

The authors reexamined the 10 articles and documented each quality improvement initiative or factor related to SNF-to-hospital readmissions and sorted them into positive facilitators or negative barriers. Broader themes were then assigned to each point (Table 2).

\section{Discussion}

The introduction of the new Medicare Inpatient Prospective Payment System decreases reimbursement to hospitals with increased readmission rates. ${ }^{11}$ For this reason, hospitals have been key forces in spurring improvements in transitions of care, quality of care, and coordination with SNFs. ${ }^{11-13}$ Although the reimbursement model does not directly incentivize SNFs to improve 30-day hospital readmission rates, improvements made to transitions of care, and quality of acute care make SNFs stand out as referral partners to hospitals. ${ }^{12,14,15}$ It is not surprising that successful strategies to reduce 30-day hospital readmissions have been advanced by hospitals and SNFs.

Sixteen facilitator themes were identified and their occurrence, frequency sum, and percent frequency are shown in Table 3. The most cited facilitator of QI initiatives that reduced readmission rates was the use of specialized staff. Successful strategies included the use of hospital pharmacists to coordinate medication reconciliation prior to transfer and during the first days following discharge. ${ }^{11}$ In fact, medication reconciliation was cited in multiple articles as a key strategy for reducing hospital readmissions. ${ }^{11,12,16,17}$ Post-discharge advocate nurses were also used by hospitals to improve transitions of care to SNFs. ${ }^{12}$ Similarly, other articles pointed to the use of physicians, mid-level providers, and advanced practice nurses to ensure proper care plans were made and orders written. ${ }^{13,17-19}$ Multiple QI initiatives put focus on patient transfer protocols that included standardized documents, checklists, order reconciliation, and post-discharge follow-up. ${ }^{11,12,16-17}$ Communication tools, such as the Situation Background Assessment Recommendation (SBAR) Method, were also cited as useful to reducing mistakes. ${ }^{14-16,20}$ Workflow improvements were made to arrange the electronic medical record documentation in a more helpful and streamlined fashion. ${ }^{11,16}$ Better coordination with specialists outside the direct hospital-SNF relationship was also noted. One study incorporated the use of neurologic telehealth consults. ${ }^{20}$ Another study used a specialized nurse working with a state quality improvement organization to provide education to facilities on proper clinical procedures and strategies aimed at QI initiatives. ${ }^{13}$ SNFs also assigned special responsibilities to existing employees, such as designating a QI champion to promote facility engagement with QI initiatives. ${ }^{15}$

Specialized staff facilitators were identified when SNFs utilized clinical supervision that did not include the standard facility employees. The clinicians discussed in the articles included pharmacists, nurse practitioners, telehealth neurologic consultants, nurse navigators, and post-discharge advocate nurses. ${ }^{11-13,15,17-20}$ Most of the QI initiatives used quality improvement models as facilitators to reduce hospital readmissions. ${ }^{12-15,18,20}$ The most cited model was the Intervention to Reduce Acute Care Transfers (INTERACT). ${ }^{13-15}$ INTERACT is a comprehensive QI program that includes educational tools and strategies designed to help empower SNF staff to properly identify, assess, document, and communicate the changing status of patients. ${ }^{13-15}$ Other QI models included the Shewhart Cycle of Plan-Do-Study-Act (STAAR), ${ }^{18}$ the Modified Brokerage Case Management Model designed to connect clients with services, ${ }^{12}$ and the use of a Collaborative Partner Prioritization Model to select the best institutions with which to work. ${ }^{20}$ Another positive facilitator was the use of collaborative case management between SNFs and hospitals either through phone calls, direct 


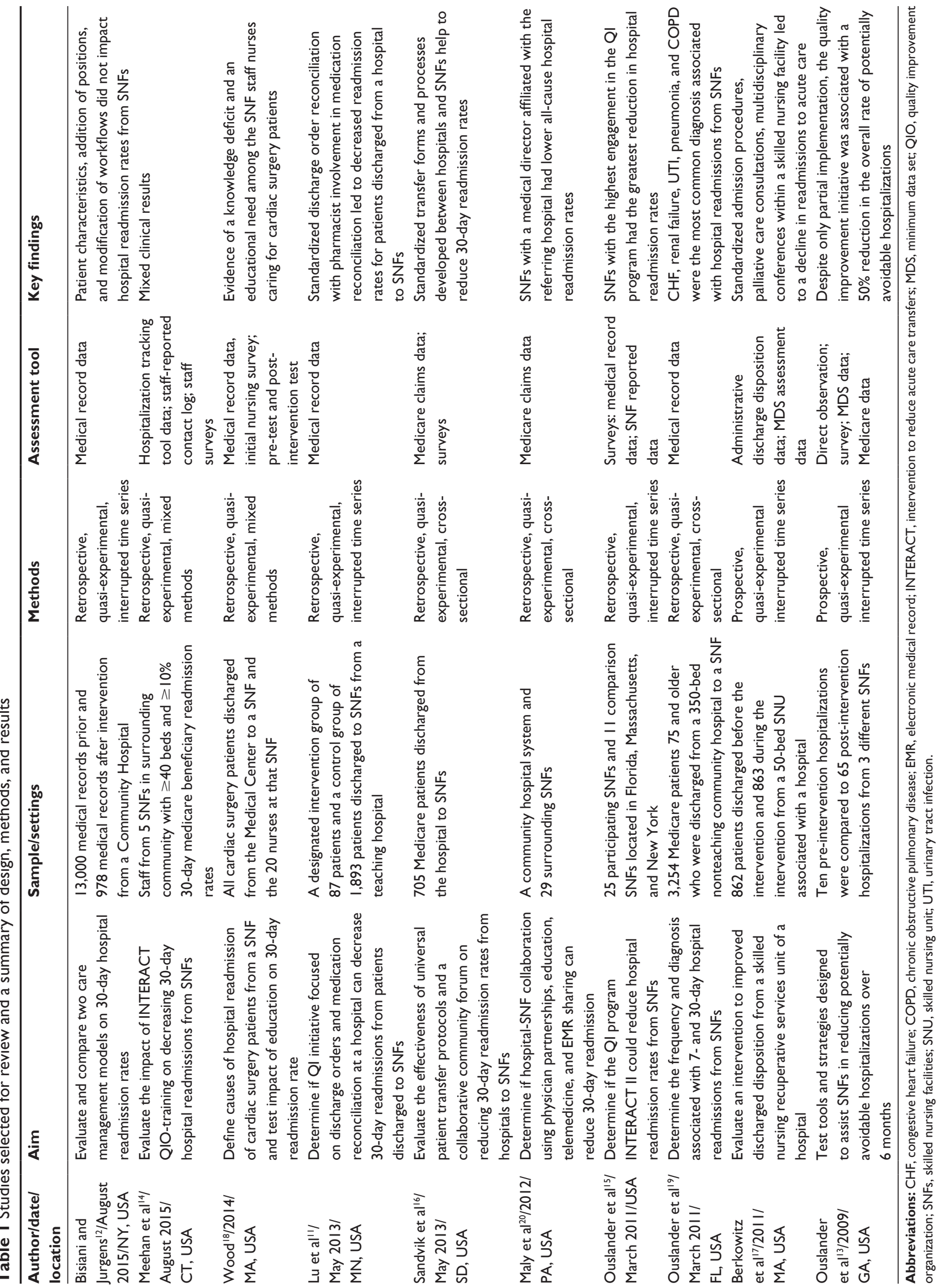


Table 2 Themes associated with quality initiatives to reduce 30-day SNF-to-hospital readmissions

\begin{tabular}{|c|c|c|}
\hline References & Positive facilitators & Negative barriers \\
\hline \multirow[t]{8}{*}{$\begin{array}{l}\text { Bisiani and } \\
\text { Jurgens }{ }^{12}\end{array}$} & I. Identification of at-risk patients can help tailor treatment & $\begin{array}{l}\text { I. Point of entry RN care manager and case. Management } \\
\text { assistants do not reduce readmission rates }\end{array}$ \\
\hline & $\begin{array}{l}\text { 2. Collaborative case management associated with lower } \\
\text { readmissions }\end{array}$ & $\begin{array}{l}\text { 2. Modification of work flows did not reduce readmission } \\
\text { rates }\end{array}$ \\
\hline & 3. Interventions saved $\$ 4 I 2$ per patient & 3. Challenge in finding experienced case managers \\
\hline & 4. Post-discharge telephone call reduces readmission & \\
\hline & 5. Use of a post-discharge advocate nurse & \\
\hline & 6. Senior leadership develop service design and strategy & \\
\hline & $\begin{array}{l}\text { 7. Medication reconciliation decreased adverse events, } \\
\text { lowers hospital readmissions }\end{array}$ & \\
\hline & 8. Use of the modified brokerage case management model & \\
\hline \multirow[t]{5}{*}{ Meehan et al ${ }^{14}$} & I. INTERACT & I. Some leaders did not see compelling reason to participate \\
\hline & 2. Conducting root cause analyses of transferred residents & $\begin{array}{l}\text { 2. Additional support needed to bring about QI initiatives, } \\
\text { not enough time }\end{array}$ \\
\hline & $\begin{array}{l}\text { 3. Providing SNF institutional information to local emergency } \\
\text { departments }\end{array}$ & 3. Not all the QI tools were implemented equally \\
\hline & 4. Leadership is critical to success of $\mathrm{QI}$ initiatives & 4. Staff lacked technologic capabilities to use hospital tracking tools \\
\hline & 5. SBAR form and progress note & \\
\hline \multirow[t]{3}{*}{ Wood $^{18}$} & $\begin{array}{l}\text { I. Use of the Shewhart cycle of plan-do-check-act and state } \\
\text { action on avoidable rehospitalizations }\end{array}$ & I. Insufficient education among SNF staff in cardiac care \\
\hline & $\begin{array}{l}\text { 2. Knowledge gap in cardiac care identified, education } \\
\text { provided in assessment, care, and interventions }\end{array}$ & $\begin{array}{l}\text { 2. Patients being readmitted for preventable or manageable } \\
\text { conditions }\end{array}$ \\
\hline & 3. Nurse practitioner involvement & 3. Only $75 \%$ of staff returned educational surveys and tests \\
\hline \multirow[t]{7}{*}{ Lu et al"I } & $\begin{array}{l}\text { I. Including pharmacists on multidisciplinary team helps } \\
\text { reduce readmission rates }\end{array}$ & $\begin{array}{l}\text { I. One study did not show significant reduction following } \\
\text { medication reconciliation, medication errors are common } \\
\text { in SNFs }\end{array}$ \\
\hline & $\begin{array}{l}\text { 2. Use of a standard discharge order reconciliation process } \\
\text { and not discharging after } 2 \mathrm{pm}\end{array}$ & $\begin{array}{l}\text { 2. Two extreme severity of illness groups did not have } \\
\text { statistical reductions in readmission rates. The illness is } \\
\text { more important than medication reconciliation }\end{array}$ \\
\hline & $\begin{array}{l}\text { 3. Medication reconciliation and } 2-4 \text { day follow up reduces } \\
\text { hospital readmissions }\end{array}$ & \\
\hline & 4. Improving electronic workflow reduced medication errors & \\
\hline & 5. Limited financial resources required for meaningful results & \\
\hline & 6. Executive leaders supported evidence-based changes & \\
\hline & $\begin{array}{l}\text { 7. Pharmacist involvement in medication reconciliation } \\
\text { reduced discrepancies }\end{array}$ & \\
\hline \multirow[t]{6}{*}{ Sandvik et al ${ }^{16}$} & $\begin{array}{l}\text { I. Geriatric community forum used to promote } \\
\text { multidisciplinary collaboration }\end{array}$ & $\begin{array}{l}\text { I. Challenges with implementation and staff engagement. Little } \\
\text { coordination between facilities, physicians, and hospitals }\end{array}$ \\
\hline & 2. Nurse-to-nurse telephone report using SBAR & 2. Low readmission rate could reflect poor-quality choices \\
\hline & $\begin{array}{l}\text { 3. Using a single set of forms with checklists during patient } \\
\text { transfer }\end{array}$ & 3. Transfer forms not always completed by sending physicians \\
\hline & $\begin{array}{l}\text { 4. Medication list with diagnosis for each medication and } \\
\text { comments helps to reduce errors }\end{array}$ & $\begin{array}{l}\text { 4. Personnel turn over causes loss of institutional knowledge } \\
\text { and continuity of QI project }\end{array}$ \\
\hline & $\begin{array}{l}\text { 5. Advanced directive form can reduce undesired } \\
\text { hospitalizations }\end{array}$ & $\begin{array}{l}\text { 5. Hospitalists with less nursing home experience overall began } \\
\text { completing transfers }\end{array}$ \\
\hline & $\begin{array}{l}\text { 6. Universal documentation with prompts, safety reminders, } \\
\text { and checklists }\end{array}$ & $\begin{array}{l}\text { 6. Improper transfer of patient data, improper documentation, } \\
\text { missing orders had been prior issue }\end{array}$ \\
\hline \multirow[t]{6}{*}{ Maly et $\mathrm{al}^{20}$} & I. Collaborative partner prioritization tool & $\begin{array}{l}\text { I. Aligning hospitals and SNFs requires like-minded leaders that } \\
\text { are willing to invest time and effort }\end{array}$ \\
\hline & 2. Use of SBAR communication tool & $\begin{array}{l}\text { 2. QI project needs time to grow in order to track and report } \\
\text { findings }\end{array}$ \\
\hline & $\begin{array}{l}\text { 3. Hospital/SNF collaboration involving patient information } \\
\text { and clinical educational resources }\end{array}$ & \\
\hline & $\begin{array}{l}\text { 4. Sharing of protocols for care of patients with congestive } \\
\text { heart failure, post-myocardial infarction, and pneumonia }\end{array}$ & \\
\hline & 5. Telehealth neurologic consults & \\
\hline & 6. Physician form for aligning goals and sharing interest & \\
\hline
\end{tabular}


Table 2 (Continued)

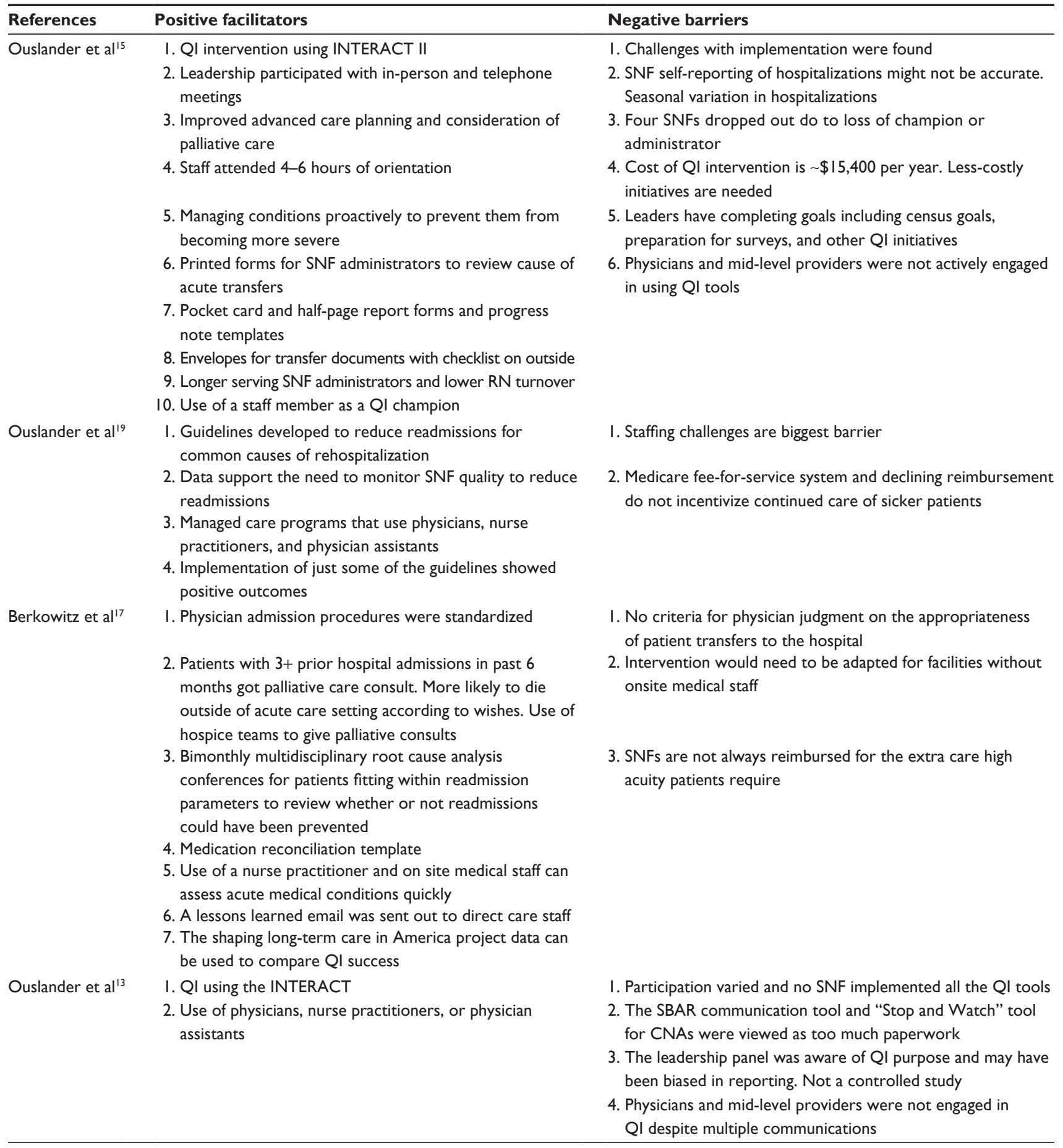

Abbreviations: CNA, certified nursing assistant; INTERACT, intervention to reduce acute care transfers; QI, quality improvement; RN, registered nurse; SBAR, situation background assessment recommendation; SNFs, skilled nursing facilities.

meetings, or geriatric community forum meetings. ${ }^{11,12,14,16,20}$ An example of collaborative case management included providing receiving emergency departments with information of SNF capabilities and procedure. ${ }^{11}$ The staff education facilitator focused on developing specialized skills for common readmitting diagnoses, proactive management of common causes of rehospitalization, ${ }^{15,18,19}$ and care paths for complex conditions, such as congestive heart failure, post-myocardial infarction, and pneumonia. ${ }^{18,20}$ Education was also used to familiarize nursing staff with the tools and protocols that were integral to the QI initiatives and to share lessons learned from root cause analysis following readmissions. ${ }^{14,15,17}$

Root cause analysis following hospital readmissions was commonly used in helping to elucidate the cause and improve 
Table 3 Facilitating themes associated with reduced 30-day skilled nursing facilities-to-hospital readmissions

\begin{tabular}{lll}
\hline Facilitator themes & $\begin{array}{l}\text { Occurrences (by article } \\
\text { number) }\end{array}$ & Sum (\%) \\
\hline $\begin{array}{l}\text { Specialized staff } \\
\text { Quality improvement model }\end{array}$ & II, I3, I4, I6, I7, I8, 19, 20 & $8(13)$ \\
$\begin{array}{l}\text { Collaborative case } \\
\text { management }\end{array}$ & $11,12,14,14,16,17,20$ & $7(11)$ \\
Care paths & $11,14,16,17,18$ & $6(10)$ \\
Leadership engagement & $11,12,14,16,17$ & $5(8)$ \\
Patient transfer protocol & $11,14,15,17,19$ & $5(8)$ \\
Communication tools & $12,15,16,17$ & $5(8)$ \\
Medication reconciliation & $11,14,15,19$ & $4(6)$ \\
Advanced and palliative care & $15,17,19$ & $4(6)$ \\
planning & & $3(5)$ \\
Quality improvement & $16,18,19$ & \\
tracking & $12,17,19$ & $3(5)$ \\
Root cause analysis & $13,17,19$ & $3(5)$ \\
Staff education & 11,14 & $3(5)$ \\
Financial resources & 17,18 & $2(3)$ \\
Implementation & 14,15 & $2(3)$ \\
Workflow & 17 & $2(3)$ \\
Staffing & & 63 \\
Total occurrences & &
\end{tabular}

QI initiatives. Many of the SNFs in this study held root cause analysis meetings in order to learn from past readmissions. ${ }^{14,15,17}$ The patient transfer protocol facilitator reflects articles that highlighted standards in transfer procedure when a hospital discharges to a SNF. Examples of transfer protocols include: the discharging hospital performing a follow-up call once a transfer has occurred, not discharging a patient to a SNF after 2 pm, and including a discharge checklist. ${ }^{11,12,15-17}$ The care paths facilitator was noted when SNFs used standard care regimes to proactively address patient populations most at risk of readmission to acute care. ${ }^{12,15,19,20}$ Multiple articles mentioned that medication reconciliation, the process of reviewing medication accuracy and appropriateness, is vital in preventing hospital readmissions. ${ }^{11,12,16,17}$

Utilization of communication tools, such as progress notes and the SBAR form had a positive impact on acute care readmission rates. ${ }^{14-16,20}$ Quality improvement tracking through the reporting and monitoring of data proved important in comparing the success of the QI initiative. ${ }^{17,19,20}$ Leadership engagement was beneficial when administrators, medical directors, and physicians took an active role in QI initiatives focused on reducing hospital admissions. Successful leaders took part in planning meetings, project design, community forums, QI implementation, root cause analysis, and goal forming and setting. ${ }^{11,12,14,15,20}$ Advanced care planning, including advanced directives and do-not-resuscitate orders, alongside an emphasis on palliative care and hospice services also helped to keep SNF residents from undesired visits to acute care. ${ }^{15-17}$
Thirteen barrier themes were identified in reference to quality improvement programs to reduce SNF-to-hospital readmissions (Table 4 ). Issues associated with quality improvement tracking and implementation were the most frequently discussed barriers to reducing 30-day SNF-tohospital readmissions. Examples of tracking issues included staff lacking the technical skills to use readmission tracking software, ${ }^{14}$ no definitive criteria for when rehospitalization was appropriate, ${ }^{13,16,17}$ the need for long-term monitoring to tell if QI initiatives were effective, ${ }^{20}$ and the need to account for seasonal differences in readmission rates. ${ }^{15}$ Specific examples of implementation barriers included lack of engagement by staff and physicians, ${ }^{15,16,18}$ partial use of available QI tools, ${ }^{13,14}$ and lack of on-site medical professionals to carry out required initiatives. ${ }^{17}$ Other barriers included the need for increased staffing and specialized personnel to carry out the improvements, both of which add expenses that are not always covered by the current, pay-for-service reimbursement model. ${ }^{15,17,19}$ Staffing issues included a general lack of personnel to carry out QI initiatives, ${ }^{14,19}$ a lack of experienced case managers, ${ }^{12}$ and a loss of QI leadership and knowledge due to turnover. ${ }^{15,16}$ Use of advanced practice and specialized staff was not always effective in improving readmission rates. Three studies showed that physicians and mid-level providers were not engaged with using QI strategies. ${ }^{13,15,16}$ Another article suggested that hospitalists were not as consistent in making quality discharges to SNFs as facility-associated physicians. ${ }^{16}$

Surveys also showed that there was inadequate training and support during and after implementation and a lack of knowledge and protocols for care paths specific to certain conditions. ${ }^{11,18}$ External factors outside the SNF's control can

Table 4 Barrier themes associated with higher 30-day skilled nursing facilities-to-hospital readmissions

\begin{tabular}{lll}
\hline Barrier themes & $\begin{array}{l}\text { Occurrences } \\
\text { (by article number) }\end{array}$ & $\begin{array}{l}\text { Sum } \\
\text { (\%) }\end{array}$ \\
\hline Implementation & $12,13,15,17,19,20$ & $6(17)$ \\
Quality improvement tracking & $12,15,16,17,19,20$ & $6(17)$ \\
Staffing & $11,12,15,17,18$ & $5(14)$ \\
Specialized staff & $11,15,17,20$ & $4(11)$ \\
Financial resources & $17,18,19$ & $3(9)$ \\
Leadership engagement & $12,15,17$ & $3(9)$ \\
Care paths & 13,14 & $2(6)$ \\
Collaborative case management & 15 & $1(3)$ \\
Communication tools & 20 & $1(3)$ \\
Medication reconciliation & 14 & $\mathrm{I}(3)$ \\
Patient transfer protocol & 15 & $\mathrm{I}(3)$ \\
Staff education & 13 & $\mathrm{I}(3)$ \\
Workflow & $\mathrm{II}$ & $\mathrm{I}(3)$ \\
Total occurrences & & 35 \\
\hline
\end{tabular}


also act as barriers to implementation, including the location of the facility, its proximity to a hospital, and the presence of state standards or local community initiatives. ${ }^{12,15,16}$ These examples help to explain why initiatives to reduce hospital readmission rates varied in their degree of success between states and communities.

A lack of leadership engagement acted as a barrier to success when leaders did not see a compelling reason to invest time and energy into QI initiatives to reduce readmissions. SNF administrators expressed that increasing census numbers and maintaining regulatory compliance were more important than reducing hospitalizations. ${ }^{14-16}$ The majority of articles highlighted the fact that leadership recognized the usefulness of a QI program to reduce readmissions but felt that employees were already taxed with heavy workloads. ${ }^{13,15,16,18,19}$ Staffing was a natural barrier in that additional staffing hours were needed to satisfy the QI initiatives, yet it was hard to find SNFs with ample and qualified staff. ${ }^{12,14-16,19}$ Lack of staff education was a noted barrier to success because staff lacked the knowledge to care for the patients most likely to be readmitted to acute care settings. ${ }^{18}$ Communication tools became barriers if certified nursing assistants and nurses in the facilities felt that forms added unnecessary paperwork. ${ }^{13}$ The financial resources needed to implement and continue a QI initiative were noted by multiple articles. ${ }^{15,17,19}$

A lack of reliable data and the time needed before seeing measurable improvements meant that quality improvement tracking could be a barrier to successful reduction in rehospitalizations. More immediate metrics to measure improvements, more accurate criteria in tracking, and less reliance on self-reporting were specific examples of tracking issues cited in studies. ${ }^{12,15-17,19,20}$ The implementation barrier was found when facilities struggled to set up quality improvement tools and adoption of procedures. ${ }^{13-17,20}$ The care paths barrier was illustrated when patients were being readmitted for manageable conditions but the staff lacked the knowledge of how to properly care for these conditions. ${ }^{11,18}$ Medication reconciliation was not a panacea to improving outcomes; medication errors were still common and did not decrease readmissions in one literature reference. ${ }^{11}$ Patient transfer protocols proved imperfect due to the failure to fully complete orders and include data when transferring between levels of care. ${ }^{16}$ Similarly, workflow redesign failed to impact the readmission rates to hospitals in another study. ${ }^{12}$

\section{Limitations}

This research review has limitations in its application. All the noted studies were conducted in a quasi-experimental manner, and most were retrospective in nature. For example, one article only considered retrospective fixed data so medical records were not accessible for root cause analysis. ${ }^{19}$ Multiple articles noted that participation in QI initiatives among SNFs varied and, in some cases facilities dropped out completely. ${ }^{12,16,19}$ The research relied mainly on smaller study samples at a state or community level. There was no consistent implementation across the nation or between nations that could be compared. ${ }^{13,15,16,19}$ Much of the research included some aspect of self-reporting, facility statistics. Self-reporting data could invite bias and open studies to errors. . $^{13,15,16}$

\section{Conclusion}

When considered from a very overarching perspective, it appears that many of the mitigating factors of the barriers presented can be overcome by facilitators which were discussed here. Allowances for the proper identification of patients and their diagnoses allows for tailored treatments to their specific condition. Collaboration in case management by teams of practitioners working with the patient will cause any barriers to care to be quickly identified, to mitigate readmissions overall. The use of post-discharge follow-up with these patients and the use of patient advocates also can be used as a tool to decrease readmissions in many circumstances. The use of quality checklist tools, such as the INTERACT/ STAAR/SBAR, and other standardized protocols will allow for a more streamlined care pipeline for patients, as these tools will allow us to provide care on a more equitable level to more patients, and these tools can be hybridized to begin to address any factors, which might be identified to be causing readmissions further reducing them as time progresses. In addition, staffing and management should be striving to provide proper education to ensure that readmissions will decrease. Any of these interventions require a strong commitment from management, so that the benefits of QII protocols are realized over time.

Hospital readmissions have become a national focus. SNFs working to find ways to reduce 30-day readmissions to acute care may consider adopting a quality improvement procedure. This study supplied a systematic review of both facilitators and barriers summarizing the results of two scholarly databases. These strategy examples can be useful to acute care hospitals attempting to lower bounce back from subacute care providers, and long-term care facilities seeking QI initiatives to reduce hospital readmissions. Focusing on a continuum of quality care results in better treatment efficiency, improved patient safety, and increased communication within partnering hospitals and SNFs. 


\section{Acknowledgments}

This article has not been published previously elsewhere in whole or in part, and it is not under consideration for publication elsewhere. It is approved by all authors, and tacitly or explicitly where the work was carried out, and that, if accepted, it will not be published elsewhere including electronically in the same form, in English or in any other language, without the written consent of the copyright-holder.

\section{Disclosure}

The authors report no conflicts of interest in this work.

\section{References}

1. Creditor MC. Hazards of hospitalization of the elderly. Ann Intern Med. 1993;118(3):219-223.

2. Mor V, Intrator O, Feng Z, Grabowski DC. The revolving door of rehospitalization from skilled nursing facilities. Health Aff (Millwood). 2010;29(1):57-64.

3. Coleman EA, Min SJ, Chomiak A, Kramer AM. Posthospital care transitions: patterns, complications, and risk identification. Health Serv Res. 2004;39(5):1449-1466

4. DeFrances CJ, Hall MJ, Podgornik MN. Advance Data From Vital and Health Statistics No 359: 2003 National Hospital Discharge Survey. Available from: http://www.cdc.gov/nchs/data/ad/ad359.pdf. Accessed September 12, 2016.

5. Donelan-McCall N, Eilertsen T, Fish R, Kramer A. Small patient population and low frequency event effects on the stability of SNF quality measures. Washington, DC, USA: Medicare Payment Advisory Commission; 2006. Available from: http://tinyurl.com/j838zb4. Accessed March 14, 2012.

6. Intrator O, Grabowski DC, Zinn J, et al. Hospitalization of nursing home residents: the effects of states' Medicaid payment and bed-hold policies. Health Serv Res. 2007;42(4):1651-1671.

7. Centers for Medicare and Medicaid Services. Medicare Nursing Home Value-Based Purchasing Initiative. 2015. Available at: https://innovation. cms.gov/initiatives/Nursing-Home-Value-Based-Purchasing/. Accessed September 21, 2016.
8. Fontanarosa PB, McNutt RA. Revisiting hospital readmissions. JAMA. 2013;309(4):398-400.

9. Berwick DM, Nolan TW, Whittington J. The triple aim: care, health, and cost. Health Aff (Millwood). 2008;27(3):759-769.

10. Guterman S, Davis K, Stremikis K, Drake H. Innovation in Medicare and Medicaid will be central to health reform's success. Health Aff (Millwood). 2010;29(6):1188-1193.

11. Lu Y, Clifford P, Bjorneby A, et al. Quality improvement through implementation of discharge order reconciliation. Am J Health Syst Pharm. 2013;70(9):815-820.

12. Bisiani MA, Jurgens CY. Do collaborative case management models decrease hospital readmission rates among high-risk patients? Prof Case Manag. 2015;20(4):188-196.

13. Ouslander JG, Perloe M, Givens JH, Kluge L, Rutland T, Lamb G. Reducing potentially avoidable hospitalizations of nursing home residents: results of a pilot quality improvement project. $J$ Am Med Dir Assoc. 2009;10(9):644-652.

14. Meehan TP, Qazi DJ, Van Hoof TJ, et al. Process evaluation of a quality improvement project to decrease hospital readmissions from skilled nursing facilities. J Am Med Dir Assoc. 2015;16(8):648-653.

15. Ouslander JG, Lamb G, Tappen R, et al. Interventions to reduce hospitalizations from nursing homes: evaluation of the INTERACT II collaborative quality improvement project. J Am Geriatr Soc. 2011; 59(4):745-753.

16. Sandvik D, Bade P, Dunham A, Hendrickson S. A hospital-to-nursing home transfer process associated with low hospital readmission rates while targeting quality of care, patient safety, and convenience: a 20-year perspective. J Am Med Dir Assoc. 2013;14(5):367-374.

17. Berkowitz RE, Jones RN, Rieder R, et al. Improving disposition outcomes for patients in a geriatric skilled nursing facility. $J$ Am Geriatr Soc. 2011;59(6):1130-1136.

18. Wood M. Improving Transitions of Care of Cardiac Surgery Patients Discharged to Skilled Nursing Facilities: A Quality Improvement Approach [doctoral dissertation]. 2014. MN, USA: Walden University.

19. Ouslander JG, Diaz S, Hain D, Tappen R. Frequency and diagnoses associated with 7-and 30-day readmission of skilled nursing facility patients to a nonteaching community hospital. J Am Med Dir Assoc. 2011;12(3):195-203.

20. Maly MB, Lawrence S, Jordan MK, et al. Prioritizing partners across the continuum. J Am Med Dir Assoc. 2012;13(9):811-816.
Clinical Interventions in Aging

\section{Publish your work in this journal}

Clinical Interventions in Aging is an international, peer-reviewed journal focusing on evidence-based reports on the value or lack thereof of treatments intended to prevent or delay the onset of maladaptive correlates of aging in human beings. This journal is indexed on PubMed Central, MedLine,

\section{Dovepress}

CAS, Scopus and the Elsevier Bibliographic databases. The manuscript management system is completely online and includes a very quick and fair peer-review system, which is all easy to use. Visit http://www.dovepress. com/testimonials.php to read real quotes from published authors. 